\title{
Temporal Rewiring of Striatal Circuits Initiated by Nicotine
}

\author{
Louise Adermark*, , Julia Morud', Amir Lotfi', Klara Danielsson', Lisa Ulenius ',2, Bo Söderpalm ${ }^{1,2}$ and \\ Mia Ericson'
}

'Addiction Biology Unit, Department of Psychiatry and Neurochemistry, Institute of Neuroscience and Physiology, The Sahlgrenska Academy at The University of Gothenburg, Gothenburg, Sweden; ²Beroendekliniken, Sahlgrenska University Hospital, Gothenburg, Sweden

\begin{abstract}
Drug addiction has been conceptualized as maladaptive recruitment of integrative circuits coursing through the striatum, facilitating drugseeking and drug-taking behavior. The aim of this study was to define temporal neuroadaptations in striatal subregions initiated by 3 weeks of intermittent nicotine exposure followed by protracted abstinence. Enhanced rearing activity was assessed in motor activity boxes as a measurement of behavioral change induced by nicotine $(0.36 \mathrm{mg} / \mathrm{kg})$, whereas electrophysiological field potential recordings were performed to evaluate treatment effects on neuronal activity. Dopamine receptor mRNA expression was quantified by qPCR, and nicotine-induced dopamine release was measured in striatal subregions using in vivo microdialysis. Golgi staining was performed to assess nicotine-induced changes in spine density of medium spiny neurons. The data presented here show that a brief period of nicotine exposure followed by abstinence leads to temporal changes in synaptic efficacy, dopamine receptor expression, and spine density in a subregion-specific manner. Nicotine may thus initiate a reorganization of striatal circuits that continues to develop despite protracted abstinence. We also show that the response to nicotine is modulated in previously exposed rats even after 6 months of abstinence. The data presented here suggests that, even though not self-administered, nicotine may produce progressive neuronal alterations in brain regions associated with goal-directed and habitual performance, which might contribute to the development of compulsive drug seeking and the increased vulnerability to relapse, which are hallmarks of drug addiction.

Neuropsychopharmacology (2016) 4I, 305 I-3059; doi:I0.1038/npp.20 I6.I I8; published online I0 August 2016
\end{abstract}

\section{INTRODUCTION}

Drug addiction is a chronic relapsing disorder characterized by compulsive drug seeking and loss of control over intake. Repeated drug taking reorganizes neural circuits, and druginduced recruitment of integrative mechanisms within the basal ganglia has been postulated to promote drug-seeking behavior (Belin et al, 2013, 2009; Yin et al, 2008). The striatal nucleus is the major input nucleus to the basal ganglia, and striatal subregions appear to be selectively recruited during specific stages of addiction. The ventral striatum, the nucleus accumbens ( $\mathrm{nAc}$ ), is associated with the rewarding and reinforcing effects by drugs of abuse ( $\mathrm{Di}$ Chiara and Imperato, 1988; Morud et al, 2015), whereas the dorsal striatum has been implicated in drug relapse (Garavan $e t$ al, 2000; Volkow et al, 2006). The dorsal striatum can be subdivided into the dorsomedial striatum (DMS), involved in drug-induced behavioral sensitization, and the dorsolateral striatum (DLS), which appears to be recruited as addiction develops into compulsive habitual drug intake (Durieux et al,

\footnotetext{
*Correspondence: Dr L Adermark, Addiction Biology Unit, Department of Psychiatry and Neurochemistry, Institute of Neuroscience and Physiology, The Sahlgrenska Academy at The University of Gothenburg, Box 410, Gothenburg 40530, Sweden, Tel: +46 31 7863975,

Fax: +46 31828 163, E-mail: louise.adermark@neuro.gu.se

Received 14 January 2016; revised 18 May 2016; accepted 30 June 2016; accepted article preview online 8 July 2016
}

2012; Gerdeman et al, 2003; Takahashi et al, 2007; Yin et al, 2005, 2006, 2008).

Previous preclinical research has identified a hierarchical recruitment of striatal subregions during the progression of cocaine use, where changes in dopamine signaling in DLS are dependent on antecedent activity in ventral striatal circuitry (Belin and Everitt, 2008; Willuhn et al, 2012). There is also dissociation between the DMS and DLS over the acquisition and performance of cocaine seeking, further supporting the theory of dynamic shifts in striatal control over cocaine seeking (Murray et al, 2012). However, temporal recruitment of dorsal striatal subregions has also been shown during consolidation of a motor skill, and neuroadaptations associated with drug self-administration in these subregions might thus be partially associated with action learning (Yin et al, 2009). If legal drugs, such as nicotine, induce similar transformations in striatal circuits, and if animals need to self-administer the drug in order for these putative changes to occur, is not known.

Nicotine, the major addictive component of tobacco, induces its reinforcing effects by activating the mesolimbic dopamine system (Di Chiara et al, 1994; Nisell et al, 1994a, b). Repeated intermittent administration of nicotine has been shown to alter nicotinic acetylcholine receptor availability, and to modulate dopaminergic neurotransmission in ways that lead to behavioral sensitization to drug exposure in a long-term manner (Adermark et al, 2015; Benwell and Balfour, 1992; Clarke et al, 1988; Le Foll et al, 2003; Morud 
et al, 2015; Vezina et al, 2007). The aim of this study was to define if a brief period of non-volitional administration of nicotine followed by protracted abstinence would be sufficient to produce temporal neuroadaptations in dorsal striatal subregions. Wistar rats were treated intermittently with nicotine for 3 weeks, and then maintained abstinent for up to 27 weeks (6 months), after which nicotine was readministered for a short time frame. Rearing activity, which is enhanced following repeated nicotine administration and impaired by lesions in the DMS (Adermark et al, 2015; Abedi Mukutenga et al, 2012), was assessed to monitor behavioral effects that could be linked to dorsal striatum function.

\section{MATERIALS AND METHODS}

\section{Nicotine Treatment}

Adult male Wistar rats (Taconic, Ejby, Denmark or Janvier, France) (250-350 g) were group-housed with a 12-h light/ dark cycle with food and water ad libitum. After 1 week of acclimatization to the animal facility, rats received daily injections of nicotine $(0.36 \mathrm{mg} / \mathrm{kg}$ subcutaneously, dissolved in $0.9 \% \mathrm{NaCl}$ with $\mathrm{pH}$ adjusted to $7.2-7.4$ with $\mathrm{NaHCO}_{3}$ ), or vehicle $(0.9 \% \mathrm{NaCl})$ on weekdays during 3 weeks, resulting in a total of 15 injections. A subset of animals was maintained for 6 months, after which they received six additional daily doses of nicotine. One group of animals received only five nicotine injections in total before being killed. All experimental protocols were approved by the Ethics Committee for Animal Experiments, Gothenburg, Sweden.

\section{Locomotor Activity}

Locomotor activity was recorded once weekly during a 3-week nicotine exposure period, as described previously (Adermark et al, 2015). In brief, following $30 \mathrm{~min}$ of habituation to the motor activity test box (Kungsbacka mät-och reglerteknik $\mathrm{AB}$, Kungsbacka, Sweden), animals received a subcutaneous injection of either nicotine or vehicle, after which locomotion was recorded for $30 \mathrm{~min}$. Horizontal activity and rearing activity was measured during the entire experiment as total infrared beam breaks, while corner time was measured in seconds. To minimize the input of contextually conditioned sensitization, animals received 11 injections in the home cage, and 4 injections in the test environment.

\section{Electrophysiology}

Brain slice preparation and field potential recordings were performed acutely (after 5 days exposure), 3 days, 1 week, 1 month (for 5 days and 3 weeks exposure), 2 months, 3 months, and 6 months after the final nicotine injection as described previously (Clarke and Adermark, 2010). The subset of animals receiving six additional doses of nicotine after 6 months of abstinence was killed 2-3 days after the last injection. Coronal brain slices $(350 \mu \mathrm{m})$ were allowed to equilibrate for $15 \mathrm{~min}$ at $30^{\circ} \mathrm{C}$, and then at least $1 \mathrm{~h}$ at room temperature in aCSF containing (in $\mathrm{mM}$ ): $124 \mathrm{NaCl}, 4.5 \mathrm{KCl}$, $2 \mathrm{CaCl}_{2}, 1 \mathrm{MgCl}_{2}, 26 \mathrm{NaHCO}_{3}, 1.2 \mathrm{NaH}_{2} \mathrm{PO}_{4}$, and $10 \mathrm{D}-$ glucose, continuously bubbled with a mixture of $95 \% \mathrm{O}_{2} / 5 \%$ $\mathrm{CO}_{2}$ gas.
For electrophysiological recordings, slices were perfused with aCSF $\left(30^{\circ} \mathrm{C}\right)$ and population spikes (PS) activated by paired-pulse stimulation (50 $\mathrm{ms}$ interpulse interval) at a frequency of $0.05 \mathrm{~Hz}$ via a monopolar tungsten electrode (World Precision Instruments, Sarasota, FL) placed at the border of the DLS and the overlying white matter, or intrastriatal in the DMS. Synaptic efficacy was evaluated by stepwise increasing afferent stimulation strength (input/ output function), and changes in release probability were estimated analyzing the paired-pulse ratio (PPR) (PS2/PS1). Slices from nicotine-treated animals were run in parallel to their corresponding vehicle-treated controls, using the same stimulation electrodes and buffers.

\section{Golgi Staining}

Nicotine-treated rats and vehicle controls were deeply anesthetized with Allfatal (100 mg/ml (pentobarbital); Omnidea AB, Stockholm, Sweden) and perfused intracardiovascular with Tyrode's solution and 4\% para-formaldehyde. Brains were impregnated in a Golgi-Cox Fast Kit according to the company protocol (FD NeuroTechnologies, Columbia, $\mathrm{MD}$ ), and cut into $60 \mu \mathrm{m}$ slices. A Zeiss lsm 700 inverted confocal microscope with a $\times 63$ oil objective (NA 1.4), and an Olympus BX60 microscope (Olympus Corp., Center Valley, PA) equipped with a $\times 100$ oil objective (NA 1.3) were used to capture images from the distal dendrites. Spine counting was carried out blind to treatment on at least two separate occasions/treatment.

\section{qPCR}

Animals were decapitated and brains submerged in ice-cold saline before dissected and frozen on dry ice. Brain tissues were homogenized in $1 \mathrm{ml}$ QIAzol Lysis Reagent (Qiagen, Hilden, Germany) using a TissueLyser LT (Qiagen) and in Qiagen's RNeasy Lipid Tissue Kit to extract total RNA. The quality and concentrations of the extracted RNA were measured using a Nanodrop2000 (Thermo Scientific, Waltham, MA). A total of $1 \mu \mathrm{g}$ RNA was converted to cDNA using the QuantiTect Reverse Transcription Kit (Qiagen). qPCRs were carried out in 96-well plates using LightCycler 480 Real-time PCR (Roche Applied Science, Penzberg, Germany). Expression levels of target genes (Drd1, Drd2, Drd3, Drd4) were normalized against reference genes RPL19 (DMS), or GAPDH and SDHA (DLS). All samples were run in duplicates, alongside with negative (water) and positive (calibrator genes) controls according to the manufacturer's instructions using a LightCycler 480 Real Time PCR System (Roche Applied Science). The data were analyzed in LightCycler 480 software, and extracted to GraphPad for further analysis. Ct values for Drd4 were $>40$ in all treatment groups, and therefore excluded from further analysis.

\section{In vivo Microdialysis}

In vivo microdialysis was performed in the nAc, DMS, and DLS of awake and freely moving Wistar rats (250-350 g) as described previously (Adermark et al, 2015; Clarke et al, 2015). In brief, rats were implanted with an I-shaped custom-made dialysis probe into the nAc (coordinates: AP: $+1.85 \mathrm{~mm}$; ML: $-1.4 \mathrm{~mm}$ relative to bregma; $\mathrm{DV}:-7.8 \mathrm{~mm}$ 
relative to brain surface), DMS $(+1.2,-2.0,-5.5)$ or DLS $(+1.2,-3.5,-5.5)$. On the day of the experiment, probes were connected to a microperfusion pump and microdialysis samples were collected every $20 \mathrm{~min}$. Dopamine was analyzed online by HPLC, and following a stable baseline animals were injected with nicotine $(0.36 \mathrm{mg} / \mathrm{kg}$ subcutaneously). Only animals with accurate probe placement were included in the data analysis.

\section{Statistical Analysis}

Data were analyzed with Clampex 10.1 (Molecular Devices, Foster City, CA) and Graph Pad Prism. Gaussian distribution was tested with D'Agostino and Pearson's omnibus normality test. All data are presented as mean values \pm SEM, and the level of significance was set to $P<0.05$. Group and treatment effects were analyzed using two-way ANOVA, and unpaired $t$-test when applicable. qPCR data were analyzed using Mann-Whitney U-test.

\section{RESULTS}

\section{Protracted Nicotine Abstinence Reveals Sustained Effects on Locomotion and Progressive Changes in Synaptic Output From Striatal Subregions}

Repeated administration of nicotine significantly enhanced horizontal activity, and suppressed corner time, in a manner that sustained even after 6 months of nicotine abstinence (vehicle $v s$ nicotine, horizontal activity: 1st injection: $t=1.15$, d.f. $=27$, $P>0.05 ;$ 15th injection: $t=5.34$, d.f. $=27, \quad P<0.001, \quad+24$ weeks abstinence: $t=6.01$, d.f. $=27, P<0.001$; corner time: 1 st injection: $t=0.91$, d.f. $=27, P>0.05 ; 15$ th injection: $t=3.44$, d.f. $=27, P<0.01,+24$ weeks abstinence: $t=4.54$, d.f. $=27$, $P<0.001$ ) (Figures $1 \mathrm{a}$ and $\mathrm{b}$ ). Nicotine significantly depressed rearing activity in naïve animals, whereas an increase in rearing activity was recorded in response to the last injection (vehicle $v s$ nicotine, 1st injection: $t=3.16$, d.f. $=27, P<0.01$; 15 th injection: $t=2.32$, d.f. $=27, P<0.05$ ). The rearing enhancing effect by nicotine was intensified following the first 6 weeks of nicotine abstinence ( +6 weeks: $t=4.26$, d.f. $=13, P<0.001$ ), but did not sustain for longer abstinence periods $(+12: t=0.77$, d.f. $=16$, $P>0.05$; $+18: t=1.23$, d.f. $=26, P>0.05 ;+24: t=1.37$, d.f. $=27$, $P>0.05$ ) (Figure 1c). In parallel to changes in locomotor activity, electrophysiological field potential recordings revealed an initial decline in DMS input/output function, indicative of reduced synaptic efficacy in this brain region of nicotine-treated rats (two-way ANOVA, 3 days: $\mathrm{F}_{(1,20)}=5.19, \quad P<0.05$; 1 week: $\mathrm{F}_{(1,22)}=8.90, P<0.01 ; 1$ month: $\mathrm{F}_{(1,19)}=8.89, P<0.01$; 2 months: $\mathrm{F}_{(1,23)}=0.16, \quad P>0.05 ; 3$ months: $\mathrm{F}_{(1,21)}=0.13$, $P>0.05$ ) (Figure 1d). Five injections of nicotine were sufficient to enhance horizontal activity and rearing (horizontal activity: $\mathrm{F}_{(1,20)}=8.39, P<0.01$; rearing activity: $\left.\mathrm{F}_{(1,20)}=10.01, P<0.01\right)$ (Figures if and g). Electrophysiological recordings performed later the same day also showed upon a similar decline in input/ output function in the DMS $\left(\mathrm{F}_{1,25}=9.83, P<0.01\right)$ (Figure $1 \mathrm{~h}$ ). This brief exposure period was, however, not sufficient to produce a long-lasting decline in DMS output (two-way ANOVA, 5 days +1 month of abstinence: $\mathrm{F}_{(1,30)}=1.27, P>0.05$ (Figure 1i).

In the DLS, on the other hand, a decline in input/output function developed over time and was significant after
3 months of nicotine abstinence (two-way ANOVA, 3 days: $\mathrm{F}_{(1,14)}=1.72, \quad P>0.05 ; \quad 1 \quad$ week: $\quad \mathrm{F}_{(1,23)}=1.52, \quad P>0.05$; 1 month: $\mathrm{F}_{(1,27)}=1.54, P>0.05 ; 2$ months: $\mathrm{F}_{(1,25)}=0.20$, $P>0.05$; 3 months: $\left.\mathrm{F}_{(1,28)}=5.54, P<0.05\right)$ (Figure 1e).

\section{Temporal Neuroadaptations in Striatal Subregions During Protracted Nicotine Abstinence}

To examine underlying causes for nicotine-induced effects on input/output function, synaptic release probability was estimated by calculating the PPR, and the number of dendritic spines was counted in Golgi-stained brain slices. $\mathrm{PPR}$, and the number of dendritic spines, was significantly increased in the DMS of nicotine-treated rats as compared with vehicle-treated controls after 1 month of nicotine abstinence (unpaired $t$-test: PPR: $t=2.65$, d.f. $=19, P<0.05$; no. of spines: $t=3.4$, d.f. $=118, P<0.001$ ) (Figure 2). Following 3 months of abstinence, however, PPR was significantly reduced, with a trend towards decreased spine density (PPR: $t=2.85$, d.f. $=28, P<0.001$; no. of spines: $t=1.71$, d.f. $=58, P=0.09$ ) (Figure 2). A parallel change was also detected when quantifying mRNA expression for the dopamine D1 receptor (D1R) and the dopamine D2 receptor (D2R). The expression was significantly enhanced in nicotine-treated rats following 1 month of abstinence (Mann-Whitney test: D1R, $P<0.05 ; \mathrm{D} 2 \mathrm{R}, P<0.05, n=10$ (vehicle), $n=11$ (nicotine)), but reduced following 3 months of abstinence (Mann-Whitney test: D1R, $P<0.05$; D2R, $P=0.11, n=13$ in both treatment groups). Dopamine D3 receptor (D3R) expression was not modulated at the time points analyzed ( 1 month: $t=0.36$, d.f. $=19, \quad P>0.05$; 3 months: $t=0.06$, d.f. $=23, P>0.05)$.

In the DLS, there were no significant effects by treatment on PPR, spine density, or mRNA expression at any timepoint analyzed ( 1 month: PPR: $t=1.35$, d.f. $=22, P>0.05$; no. of spines: $t=1.87$, d.f. $=116, P=0.06$; D1R expression: $P>0.05 ; \quad$ D2R expression: $P>0.05 ;$ D3R expression: $t=0.64$, d.f. $=18, P>0.05 ; 3$ months: PPR: $t=0.36$, d.f. $=14$, $P>0.05$; no. of spines: $t=0.06$, d.f. $=60, P>0.05 ; \mathrm{D} 1 \mathrm{R}$ expression: $P>0.05$; D2R expression: $P>0.05$; D3R expression: $P>0.05$ ) (Figure 2).

\section{Nicotine Enhances In Vivo Dopamine Levels in a Subregion-Selective Manner}

Dopamine receptor expression was selectively altered in the DMS, which may relate to subregion-specific effects by nicotine on dopamine release. The extracellular concentrations of dopamine were measured by means of in vivo microdialysis in the DLS, DMS, or nAc of awake and freely moving rats during acute administration of nicotine $(0.36 \mathrm{mg} / \mathrm{kg}$ subcutaneously). Nicotine-induced dopamine release in nicotine-naïve rats was significantly higher in the nAc as compared with DMS and DLS $\left(\mathrm{F}_{(2,18)}=26, P<0.001\right)$, indicating that nicotine enhances dopamine levels in a subregion-specific manner (Figure 3a). Similar findings were retrieved following 3 weeks of repeated intermittent administration of nicotine $\left(\mathrm{F}_{(2,20)}=13, P<0.001\right)$. The relative release of dopamine in response to nicotine administration in the DMS was not significantly higher as compared with the $\operatorname{DLS}\left(\mathrm{F}_{(1,15)}=1.68, P>0.05\right)$ (Figure $\left.3 \mathrm{~b}\right)$. 

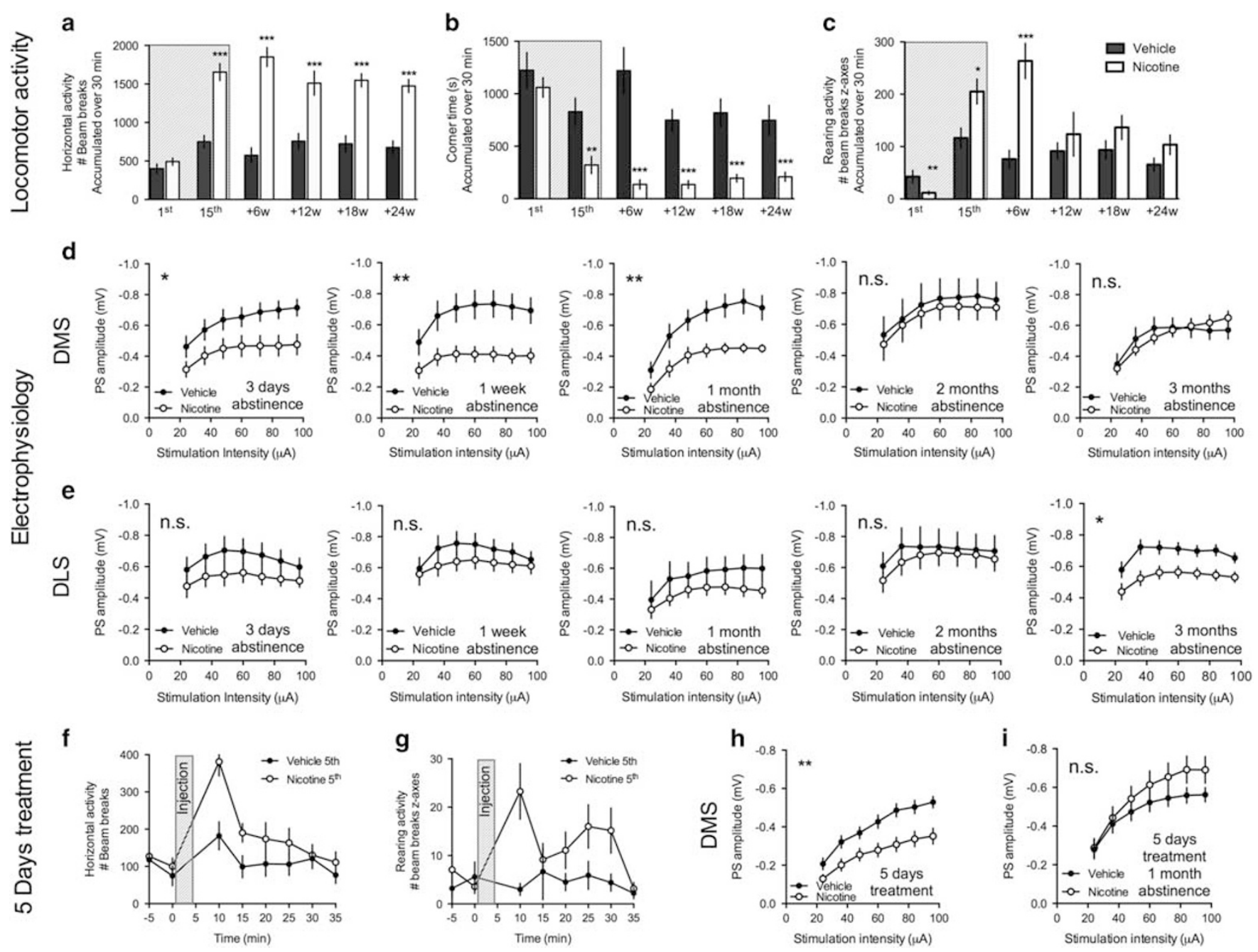

Figure I Long-lasting changes in locomotion and progressive changes in striatal neurotransmission following nicotine exposure. Rats received I 5 injections of either vehicle or nicotine over a 3-week period (hatched area) and locomotor activity in response to vehicle or nicotine injection was assessed in different batches of animals after $6,12,18$, and 24 weeks of abstinence ( $n=6-12$ per group). ( $a$ and b) Repeated administration of nicotine significantly enhance horizontal activity and suppressed comer time in a manner that was sustained even after 6 months of nicotine abstinence. (c) Nicotine initially depressed rearing activity, whereas repeated administration enhanced vertical beam breaks for up to 6 weeks after the last administration. (d) Previous exposure to nicotine significantly depressed the response amplitude in the DMS for up to I month. (e) In the dorsolateral striatum (DLS), a decline in input/output function developed over time and was significant following 3 months of nicotine abstinence. ( $f$ and g) Five injections of nicotine were sufficient to enhance horizontal activity and rearing. Time-course figure shows beam breaks during the 5 th administration of vehicle/nicotine $(n=10$ per group). (h) Input/output function was significantly depressed in the DMS of rats receiving five injections of nicotine as compared with vehicle-treated control. (i) This treatment paradigm, however, was not sufficient to produce a long-lasting decline in population spikes (PS) amplitude during nicotine abstinence. *Significant effect by treatment (*P $<0.05$, $* * P<0.01$, and $* * * P<0.001)$.

\section{Enhanced Responsiveness to Nicotine in the DLS After 6 Months of Nicotine Abstinence}

To assess long-lasting changes in neurotransmission and drug reinforcement caused by previous exposure, vehicleand nicotine-treated rats were maintained abstinent for 6 months before brain slice preparation. Electrophysiological recordings at this time-point showed no effect by treatment, neither on input/output function nor on PPR in any subregion studied (DMS: $\mathrm{I} / \mathrm{O}, \mathrm{F}=0.11_{(1,41)}, P>0.05$; PPR, $P=0.74, t=0.33$, d.f. $=22$; DLS: $\mathrm{I} / \mathrm{O}, \mathrm{F}=0.03_{(1,60)}, P>0.05$; PPR, $P=0.59, t=0.55$, d.f. $=59$ ) (Figure 4$)$.

To evaluate possible changes in the sensitivity to nicotine re-exposure, a subset of animals received six additional nicotine injections before being killed. Nicotine enhanced horizontal activity and rearing activity to a greater extent in rats receiving nicotine 6 months earlier (horizontal activity: $t=3.33$, d.f. $=14, P<0.01$; rearing activity: $t=3.05$, d.f. $=14$, $P<0.01$ ). Following 5 days of additional treatments, horizontal activity continued to be more pronounced in rats previously treated with nicotine (6th injection: $t=2.18$, d.f. $=14, P<0.05$ ), while there was no difference with respect to rearing activity (6th injection: $t=0.96$, d.f. $=14, P>0.05$ ) (Figure 4a). After 6 days of nicotine treatment to both vehicle- (vehicle+6) and nicotine-treated rats (nicotine+6) field potential recordings revealed no treatment effect on input/output function in the DMS $\left(\mathrm{F}=2.63_{(1,18)}, P>0.05\right)$, while striatal output from the DLS was significantly depressed in rats re-exposed to nicotine $\left(\mathrm{F}=5.83_{(1,17)}\right.$, $P<0.05$ ) (Figure 4). PPR was not significantly modulated 


\begin{tabular}{|c|c|c|c|c|c|c|}
\hline \multicolumn{3}{|c|}{3 weeks treatment } & \multirow{2}{*}{$\begin{array}{l}1 \text { month } \\
\text { abstinence }\end{array}$} & \multirow{2}{*}{$\begin{array}{l}\text { Golgi staining } \\
\text { Electrophysiology } \\
\text { qPCR }\end{array}$} & \multirow{2}{*}{$\begin{array}{l}3 \text { months } \\
\text { abstinence }\end{array}$} & \multirow{2}{*}{$\begin{array}{l}\text { Golgi staining } \\
\text { Electrophysiology } \\
\text { qPCR }\end{array}$} \\
\hline \begin{tabular}{|l|} 
Vehicle/ \\
nicotine
\end{tabular} & $\begin{array}{l}\text { Vehicle/ } \\
\text { nicotine }\end{array}$ & \begin{tabular}{|l|} 
Vehicle/ \\
nicotine
\end{tabular} & & & & \\
\hline
\end{tabular}

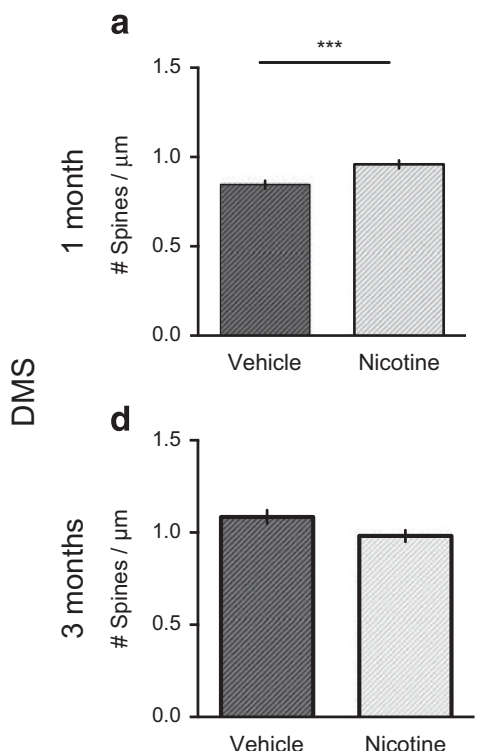

b
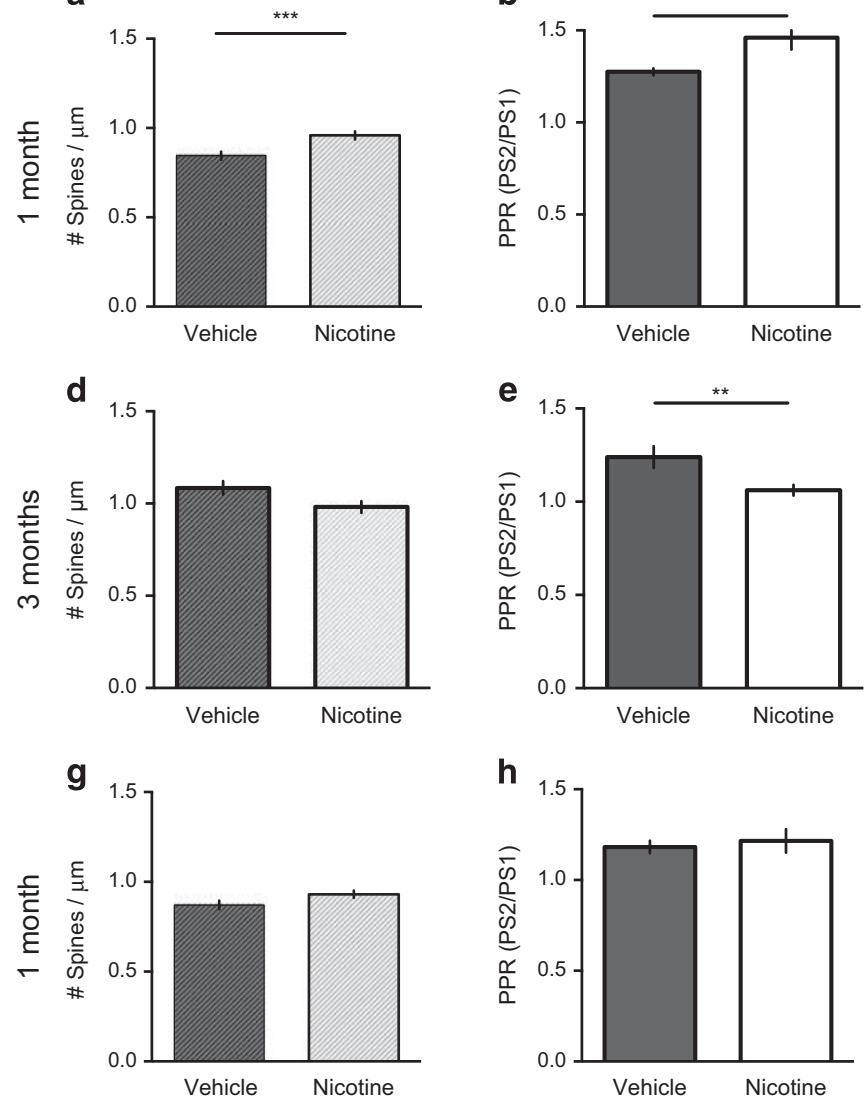

$\stackrel{\infty}{0}$

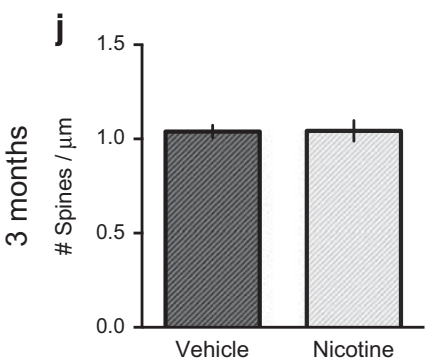

h

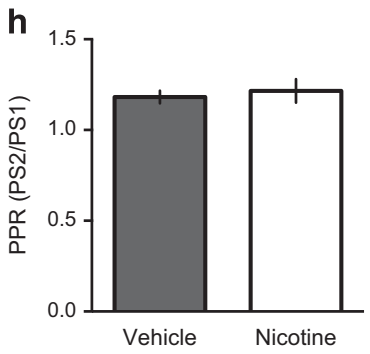

k

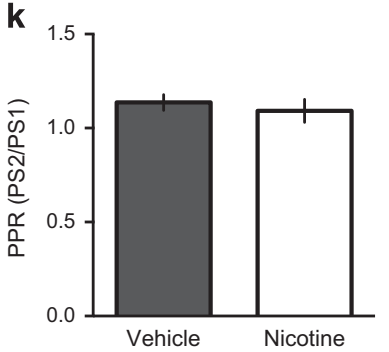

C

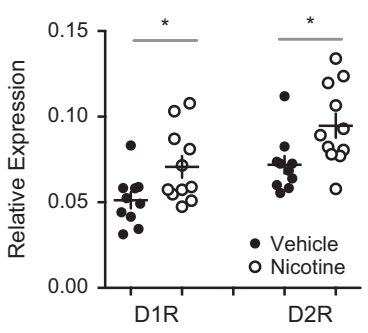

f

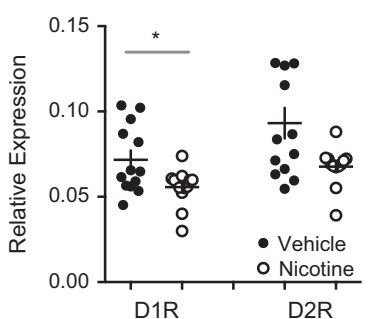

i
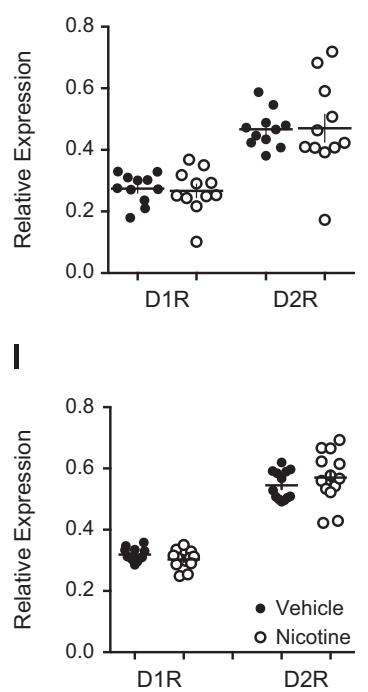

Figure 2 Nicotine abstinence produces temporal neuroadaptations in specific subregions. (a and b) Following I month of withdrawal, spine density and paired-pulse ratio (PPR) was enhanced in the dorsomedial striatum (DMS) of nicotine-treated rats, indicative of a decrease in probability for transmitter release but increase in the number of synapses in this treatment group. (c) In addition, both dopamine DI and D2 receptor mRNA expressions were enhanced in nicotine-treated rats. ( $\mathrm{d}-\mathrm{f})$ After 2 additional months of abstinence, treatment effects on spine density, PPR, and mRNA expression were reversed in the DMS. $(g-l)$ In the dorsolateral striatum (DLS), spine density, PPR, or mRNA expression were not significantly modulated following neither I nor 3 months of abstinence. *Significant effect by treatment $(* P<0.05$, **P $<0.01$, and $* * * P<0.001)$.

in any subregion (vehicle+6 vs nicotine+6: DMS: $t=0.06$, d.f. $=11, P>0.05 ;$ DLS: $t=1.27$, d.f. $=11, P>0.05)$, while spine density was enhanced in rats previously exposed to nicotine in both subregions (vehicle $v s$ nicotine+6: DMS: $t=3.3$, d.f. $=13 ; P<0.01$, DLS: $t=2.50$, d.f. $=16, P<0.05$ ) (Figure 4). In addition, in the DMS, spine density was significantly enhanced in previously exposed rats as compared with vehicle-treated rats receiving nicotine for the first time (vehicle+6 vs nicotine+6: DMS: $t=3.1$, d.f. $=10$; $P<0.05$; DLS: $t=1.4$, d.f. $=12, P>0.05$ ) (Figure 4).

\section{DISCUSSION}

Drug addiction is a chronic relapsing disorder that appears to be related to progressive neuroadaptations of corticostriatal networks (Belin et al, 2008). Previous studies have shown that striatal subregions are recruited in a hierarchical manner during the progression of cocaine use, and the data presented here suggest that similar processes might occur in response to nicotine treatment. Three weeks of intermittent nicotine administration was sufficient to produce long- 
a

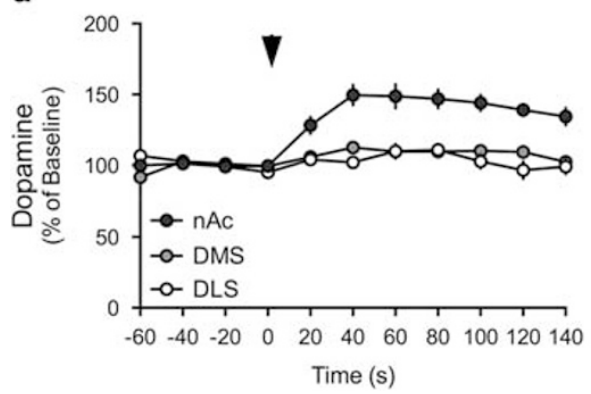

b

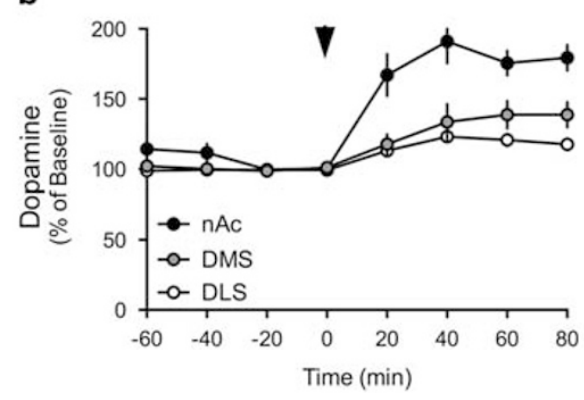

C

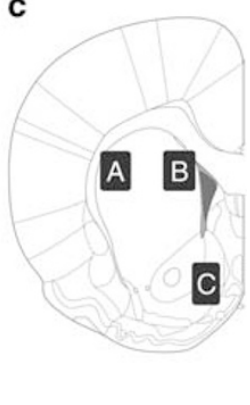

Figure 3 Nicotine enhances extracellular dopamine levels in a subregion-specific manner. (a) Administration of nicotine selectively enhanced the extracellular concentration of dopamine in the nucleus accumbens $(n A c)$ of nicotine-naive rats. (b) The subregion-specific release of dopamine remained in rats subjected to 3 weeks of intermittent nicotine administration. Arrow marks time point for nicotine administration ( $0.36 \mathrm{mg} / \mathrm{kg}$, subcutaneously). (c) Schematic drawing describing the areas of microdialysis probe placement in the different subregions of the striatum: (A) DLS, (B) DMS, and (C) nAc.

a

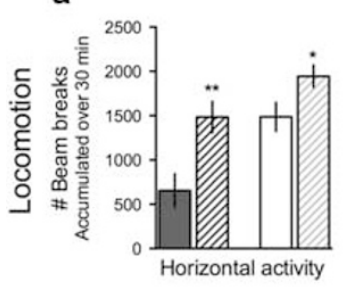

C

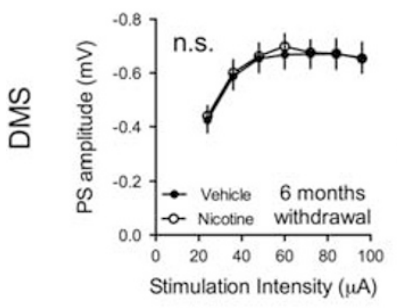

h

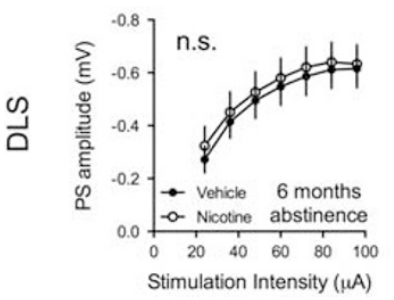

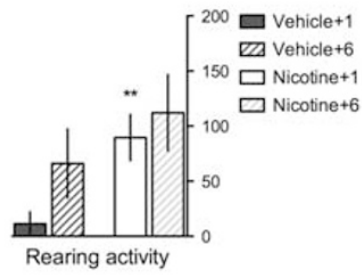

d

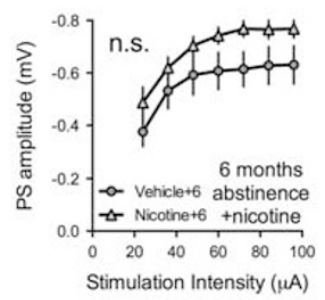

i

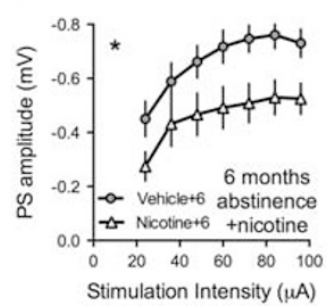

b
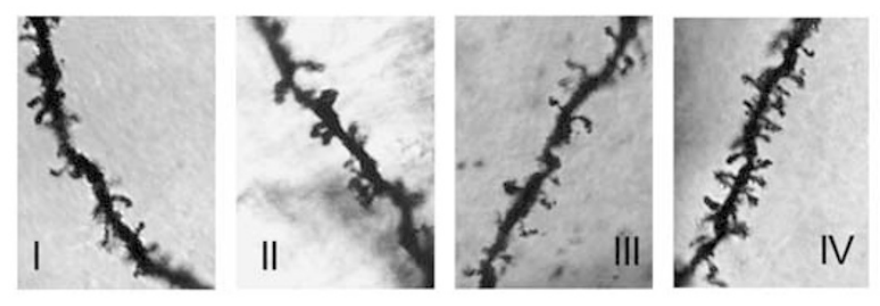

e

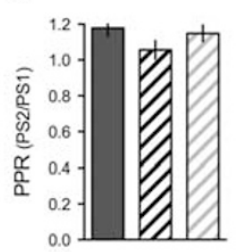

$f$

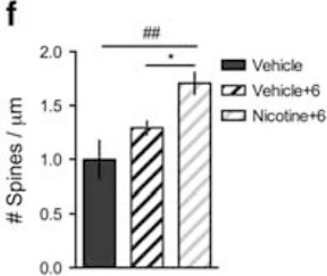

g

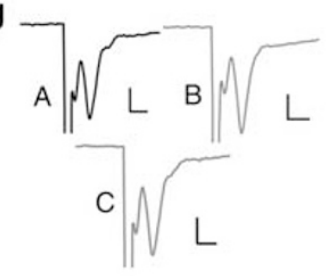

j

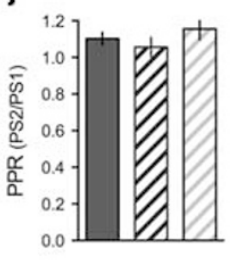

k

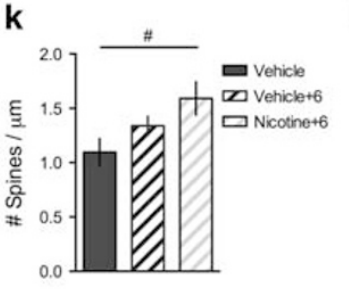

I

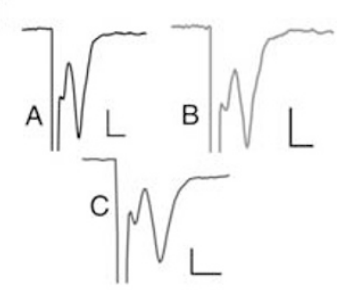

Figure 4 Changes in responsiveness to nicotine after 6 months of nicotine abstinence. After 6 months of nicotine abstinence, vehicle- (vehicle+6) and nicotine-treated (nicotine+6) rats received 6 days of nicotine administration. (a) Horizontal activity and rearing activity were significantly enhanced in response to the first nicotine injection in rats previously exposed to nicotine as compared with vehicle-treated control rats housed in parallel. (b) Micrographs showing representative Golgi staining of dendritic spines in the dorsomedial striatum (DMS); vehicle (I), vehicle receiving nicotine (vehicle+6) (II), nicotine (III), and nicotine receiving nicotine (nicotine+6) (IV). (c) Input/output function was restored in the DMS after 6 months of nicotine abstinence. (d) Re-exposure to nicotine did not depress population spikes (PS) amplitude in the DMS of rats previously exposed to nicotine. (e) Paired-pulse ratio (PPR) was not significantly modulated by treatment in the DMS. ( $f$ ) Spine density was enhanced by nicotine in the DMS of rats previously exposed to nicotine (nicotine+6). (g) Example traces showing evoked PS amplitudes in the DMS of slices from vehicle (A), nicotine (B), and nicotine+6-treated rats (C). (h) Input/output function was not modulated in the dorsolateral striatum (DLS) following 6 months of abstinence. (i) Six additional days of nicotine treatment significantly depressed input/ output function in rats previously treated with nicotine (nicotine+6). (j) PPR was not modulated by treatment in the DLS. (k) Nicotine exposure significantly enhanced DLS spine density in re-exposed rats. (I) Example traces showing evoked PS amplitudes in the DLS of slices from vehicle (A), nicotine (B), and nicotine+6-treated rats (C). Calibration is $0.15 \mathrm{mV}, 0.2 \mathrm{~ms}$. *Significant effect by previous treatment $\left({ }^{*} P<0.05\right.$ and $\left.{ }^{* *} P<0.0 \mathrm{I}\right)$. ${ }^{\#}$ Significant effect compared with vehicle ( ${ }^{\#} P<0.05$ and $\left.{ }^{\# \#} P<0.01\right)$.

lasting changes in synaptic efficacy that developed over time in a subregion-selective manner. Five days of intermittent nicotine exposure was sufficient to produce a robust decline in DMS input/output function, while significant changes in
DLS was established after several months of abstinence, at a time point where DMS activity was restored. These findings thereby suggest that a brief period of intermittent nicotine treatment might be sufficient to instigate rewiring of 
neuronal networks that may continue to develop during protracted abstinence. In addition, even though treatment effects on synaptic transmission appeared to be fully recovered after 6 months of nicotine abstinence, brief re-exposure rapidly reinstated alterations in synaptic efficacy in the DLS, which, after the initial treatment period, required several months to develop. Notably, 5 days of intermittent nicotine treatment was not sufficient to produce long-lasting changes in DMS activity, suggesting that the persistence of these adaptations may depend on the number of exposures and/or the total dose received (Ksir et al, 1987; Schoffelmeer et al, 2002). It should be noted, however, that nicotine was administered in a non-volitional manner, which might not recruit the same neuronal circuits as drug selfadministration. It is thus possible that voluntary consumption further strengthens neuroadaptations in corticostriatal networks, and that a shorter drug use could be sufficient to induce sustained changes in neurotransmission.

Previous research indicates that the DLS and DMS have separable roles in the process of behavioral sensitization and in the development of addictive behavior. The DMS, but not DLS, appears to be recruited during cocaine-induced behavioral sensitization (Durieux et al, 2012; Kim et al, 2013), and dopaminergic manipulations in the DMS reduce cocaine-seeking behavior in its early stages, while infusion of dopamine antagonists into the DLS decreases established cocaine-seeking behavior (Murray et al, 2012). A similar modulation of striatal neurotransmission has been shown during acquisition and consolidation of a motor skill, where inputs from the associative cortex are strengthened at an early state, while inputs from the sensory motor cortex are gradually potentiated over time (Yin et al, 2009). Interestingly, dopamine systems also adapt during acquisition and consolidation of a motor skill, where initial changes in dopamine D1R expression in DMS progressively decline with consolidation (Sommer et al, 2014). Dorsal striatal neuroadaptations monitored over a period of drug selfadministration might thus be partially influenced by action learning. Thus, importantly, the data presented here indicate that non-volitional nicotine administration recruits similar cortical areas and initiates a comparable transition, suggesting that these progressive effects on neurotransmission in the DMS and DLS follow a specific activation pattern of cortical subregions that may not require motor skill learning (Voorn et al, 2004). Importantly, excitotoxic lesions in the DMS selectively suppress motor skill learning during the early, but not late, phase, indicating that these processes develop in parallel and that neuroadaptations in the DLS may occur independent of changes in the DMS (Yin et al, 2009).

Dopamine is a key neurotransmitter for the reinforcing properties of drugs of abuse, and changes in dopamine sensitivity have been linked to the switch from actionoutcome responding into abnormal habit responding, leading to incentive drug seeking and craving (Di Chiara, 2000). The data presented here show subregion-selective changes in mRNA expression of both dopamine D1Rs and D2Rs in the DMS, but not in DLS, indicating that nicotine withdrawal produces temporal adaptations in dopaminergic transmission. In addition, a role for dopamine in the neuroadaptations presented here is further supported by changes in spine density, which was selectively enhanced in the DMS during the initial phase of drug treatment.
Increased spine density has been associated with dopaminedependent structural plasticity (Dietz et al, 2012; Meredith et al, 1995; Ren et al, 2010; Wickens et al, 2003), and appears to be especially promoted by dopamine D1R activation (Kozorovitskiy et al, 2015). D3Rs and D4Rs have also been implicated in behavioral sensitization and nicotine-seeking behavior (Guillin et al, 2001; Yan et al, 2012). However, D3R mRNA levels were not significantly modulated at the time points analyzed, and the expression level of $\mathrm{D} 4 \mathrm{R}$ was too low to reach inclusion in either treatment group.

Importantly, even though nicotine activates the mesolimbic dopamine system (Adermark et al, 2015; Grenhoff et al, 1986; Nisell et al, 1994b; Pontieri et al, 1996), and has been reported to enhance dopamine levels in the dorsal striatum (Marshall et al, 1997), this is not always the case for the putamen (Gallezot et al, 2014). The in vivo microdialysis performed here displayed a substantially lower dopamine increase in rat dorsal striatum as compared with the nAc. This differential effect sustained even after 3 weeks of nicotine administration, and did not support a significant deviation between the DMS and DLS. In addition, previous data from our laboratory has shown that input/output function is not significantly modulated in the nAc of adult rats following 3 weeks of nicotine treatment (Adermark et al, 2015). The acute neuroadaptations detected in the DMS are thus not likely initiated by changes in dopamine transmission, even though changes in this system appears to occur during the progression of nicotine withdrawal. Rather, these neuroadaptations in the dorsal striatum might be connected to activation/deactivation of specific cortical areas and inputs reaching the DMS and DLS, or via local nicotine effects (Falasca et al, 2014; Konradsson-Geuken et al, 2009).

Repeated intermittent administration of nicotine increased horizontal activity and rearing activity, and suppressed the time spent in corners in a long-lasting manner. Interestingly, enhanced rearing activity sustained for up to 6 weeks of abstinence, and is therefore not equivalent to behavioral sensitization to the locomotor stimulatory properties of nicotine, which appears to be life long (Morud et al, 2015). Even though no causal relationship can be established here, changes in rearing activity appeared to correlate with suppressed neurotransmission in the DMS. In fact, the DMS has previously been implicated in rearing-, but not locomotor, activity (Abedi Mukutenga et al, 2012), suggesting that separate neurocircuits may underlie these behavioral effects of nicotine. It should be noted, however, that when readministering nicotine after 6 months of abstinence, the rearing activity was initially higher in rats previously exposed to the drug. This indicates that tolerance to the rearing suppressing properties of nicotine sustains even after 6 months of nicotine abstinence.

Treatment effects on synaptic efficacy were assessed by stepwise increasing stimulation strength, and the data presented here suggest that excitatory neurotransmission is selectively declined in the DMS of rats that have received nicotine. The depression was concomitant with a change in $\mathrm{PPR}$, indicating that the probability for transmitter release is selectively decreased in the DMS after 1 month of nicotine abstinence. Surprisingly, in parallel to this decline in synaptic efficacy, the number of dendritic spines was increased. This finding, however, might be associated with an increased number of silent synapses, which has previously been shown 
during cocaine-induced behavioral sensitization (Brown et al, 2011). In the DLS, input/output function was significantly depressed first 3 months after the last nicotine administration. At the same time, the DLS responded more robustly to nicotine re-exposure after 6 months of protracted abstinence. This finding supports pervious theories of a dynamic shift between brain regions associated with goaldirected and habitual performance during the progression of drug addiction (Gremel and Costa, 2013; Murray et al, 2012), and is also in line with the concept that neuroadaptations initially occurring in the DMS are in some way transferred to the DLS where they become permanent (Belin et al, 2008; Miyachi et al, 2002; Takahashi et al, 2007). The data presented here, however, is purely descriptive in nature, and casual relationships need to be further established before we can determine if these effects are relevant for nicotine addiction.

Previous research suggests that protracted exposure to addictive drugs recruit dopamine-dependent, striatonigrostriatal ascending spirals from the nAc to more dorsal regions of the striatum, and that these transformations underlie a shift from action-outcome to stimulus-response mechanisms in the control over drug seeking (Belin et al, 2009; BelinRauscent et al, 2012; Yin et al, 2008). Our data suggest that nicotine may recruit similar pathways and produce comparable reorganizations of striatal circuits. Importantly, the data presented here are based on forced drug exposure and do not involve self-administration or instrumental conditioning. Thus, passive exposure to drugs of abuse may not only activate these pathways but may also produce prolonged reorganizations independent of administration or motivational aspects of drug taking. In addition, we show that longlasting changes in striatal connectivity may develop during protracted abstinence, and that these neuromodulations may render the system vulnerable to further exposure to the drug. Considering the role of DLS in habit formation, this shift in dorsal striatal circuits could be involved in the development of compulsive drug seeking and the high vulnerability to relapse, which are hallmarks of drug addiction.

\section{FUNDING AND DISCLOSURE}

The authors declare no conflict of interest.

\section{ACKNOWLEDGMENTS}

We acknowledge Rosita Stomberg for assisting during behavioral sensitization, microdialysis, fixation for Golgi staining, and HPLC analysis, Maria Mårdh and Mehrnoush Mohammad Davoudi for assisting during the electrophysiological recordings, Maria Kinnander for assisting during behavioral nicotine sensitization, Marta Perez Alcazar and Vilhelm Adermark for counting dendritic spines, and Erik Thurin for assisting during in vivo microdialysis. We also thank the Center of Cellular Imaging at Sahlgrenska Academy, University of Gothenburg, for the opportunity to use their imaging equipment and the support from the staff. This work was supported by the Stiftelsen Psykiatriska Forskningsfonden, Swedish Brain Foundation, the Swedish Medical Research Council (Diary numbers 2014-3888, 2014-3887, and 2015-02894), Åhlén-stiftelsen, Åke Wibergs foundation, Bror Gadelius Minnesfond, governmental

support under the LUA/ALF agreement, Wilhelm och Martina Lundgrens vetenskapsfond, and Magnus Bergvall's Foundation.

\section{REFERENCES}

Abedi Mukutenga P, Taghzouti K, Bengelloun WA (2012). Effects of bilateral electrolytic lesions of the dorsomedial striatum on motor behavior and spatial learning in rats. Basic Clin Neurosci 3: 52-59.

Adermark L, Morud J, Lotfi A, Jonsson S, Soderpalm B, Ericson M (2015). Age-contingent influence over accumbal neurotransmission and the locomotor stimulatory response to acute and repeated administration of nicotine in Wistar rats. Neuropharmacology 97: 104-112.

Belin D, Belin-Rauscent A, Murray JE, Everitt BJ (2013). Addiction: failure of control over maladaptive incentive habits. Curr Opin Neurobiol 23: 564-572.

Belin D, Everitt BJ (2008). Cocaine seeking habits depend upon dopamine-dependent serial connectivity linking the ventral with the dorsal striatum. Neuron 57: 432-441.

Belin D, Jonkman S, Dickinson A, Robbins TW, Everitt BJ (2009). Parallel and interactive learning processes within the basal ganglia: relevance for the understanding of addiction. Behav Brain Res 199: 89-102.

Belin-Rauscent A, Everitt BJ, Belin D (2012). Intrastriatal shifts mediate the transition from drug-seeking actions to habits. Biol Psychiatry 72: 343-345.

Benwell ME, Balfour DJ (1992). The effects of acute and repeated nicotine treatment on nucleus accumbens dopamine and locomotor activity. Br J Pharmacol 105: 849-856.

Brown TE, Lee BR, Mu P, Ferguson D, Dietz D, Ohnishi YN et al (2011). A silent synapse-based mechanism for cocaine-induced locomotor sensitization. J Neurosci 31: 8163-8174.

Clarke PB, Fu DS, Jakubovic A, Fibiger HC (1988). Evidence that mesolimbic dopaminergic activation underlies the locomotor stimulant action of nicotine in rats. J Pharmacol Exp Ther 246: 701-708.

Clarke RB, Adermark L (2010). Acute ethanol treatment prevents endocannabinoid-mediated long-lasting disinhibition of striatal output. Neuropharmacology 58: 799-805.

Clarke RB, Soderpalm B, Lotfi A, Ericson M, Adermark L (2015). Involvement of inhibitory receptors in modulating dopamine signaling and synaptic activity following acute ethanol exposure in striatal subregions. Alcohol Clin Exp Res 39: 2364-2374.

Di Chiara G (2000). Role of dopamine in the behavioural actions of nicotine related to addiction. Eur J Pharmacol 393: 295-314.

Di Chiara G, Imperato A (1988). Drugs abused by humans preferentially increase synaptic dopamine concentrations in the mesolimbic system of freely moving rats. Proc Natl Acad Sci USA 85: 5274-5278.

Di Chiara G, Morelli M, Consolo S (1994). Modulatory functions of neurotransmitters in the striatum: $\mathrm{ACh} /$ dopamine/NMDA interactions. Trends Neurosci 17: 228-233.

Dietz DM, Sun H, Lobo MK, Cahill ME, Chadwick B, Gao V et al (2012). Rac1 is essential in cocaine-induced structural plasticity of nucleus accumbens neurons. Nat Neurosci 15: 891-896.

Durieux PF, Schiffmann SN, de Kerchove d'Exaerde A (2012). Differential regulation of motor control and response to dopaminergic drugs by $\mathrm{D} 1 \mathrm{R}$ and $\mathrm{D} 2 \mathrm{R}$ neurons in distinct dorsal striatum subregions. EMBO J 31: 640-653.

Falasca S, Ranc V, Petruzziello F, Khani A, Kretz R, Zhang X et al (2014). Altered neurochemical levels in the rat brain following chronic nicotine treatment. J Chem Neuroanat 59-60: 29-35.

Gallezot JD, Kloczynski T, Weinzimmer D, Labaree D, Zheng MQ, Lim $\mathrm{K}$ et al (2014). Imaging nicotine- and amphetamine-induced dopamine release in rhesus monkeys with [(11)C]PHNO vs [(11) C]raclopride PET. Neuropsychopharmacology 39: 866-874. 
Garavan H, Pankiewicz J, Bloom A, Cho JK, Sperry L, Ross TJ et al (2000). Cue-induced cocaine craving: neuroanatomical specificity for drug users and drug stimuli. Am J Psychiatry 157: 1789-1798.

Gerdeman GL, Partridge JG, Lupica CR, Lovinger DM (2003). It could be habit forming: drugs of abuse and striatal synaptic plasticity. Trends Neurosci 26: 184-192.

Gremel CM, Costa RM (2013). Orbitofrontal and striatal circuits dynamically encode the shift between goal-directed and habitual actions. Nat Commun 4: 2264.

Grenhoff J, Aston-Jones G, Svensson TH (1986). Nicotinic effects on the firing pattern of midbrain dopamine neurons. Acta Physiol Scand 128: 351-358.

Guillin O, Diaz J, Carroll P, Griffon N, Schwartz JC, Sokoloff P (2001). BDNF controls dopamine D3 receptor expression and triggers behavioural sensitization. Nature 411: 86-89.

Kim M, Kim W, Baik JH, Yoon BJ (2013). Different locomotor sensitization responses to repeated cocaine injections are associated with differential phosphorylation of GluA1 in the dorsomedial striatum of adult rats. Behav Brain Res 257: 71-76.

Konradsson-Geuken A, Gash CR, Alexander K, Pomerleau F, Huettl P, Gerhardt GA et al (2009). Second-by-second analysis of alpha 7 nicotine receptor regulation of glutamate release in the prefrontal cortex of awake rats. Synapse 63: 1069-1082.

Kozorovitskiy Y, Peixoto R, Wang W, Saunders A, Sabatini BL (2015). Neuromodulation of excitatory synaptogenesis in striatal development. eLife 4: e10111.

Ksir C, Hakan RL, Kellar KJ (1987). Chronic nicotine and locomotor activity: influences of exposure dose and test dose. Psychopharmacology 92: 25-29.

Le Foll B, Diaz J, Sokoloff P (2003). Increased dopamine D3 receptor expression accompanying behavioral sensitization to nicotine in rats. Synapse 47: 176-183.

Marshall DL, Redfern PH, Wonnacott S (1997). Presynaptic nicotinic modulation of dopamine release in the three ascending pathways studied by in vivo microdialysis: comparison of naive and chronic nicotine-treated rats. J Neurochem 68: 1511-1519.

Meredith GE, Ypma P, Zahm DS (1995). Effects of dopamine depletion on the morphology of medium spiny neurons in the shell and core of the rat nucleus accumbens. J Neurosci 15(5 Pt 2): 3808-3820.

Miyachi S, Hikosaka O, Lu X (2002). Differential activation of monkey striatal neurons in the early and late stages of procedural learning. Exp Brain Res 146: 122-126.

Morud J, Adermark L, Perez-Alcazar M, Ericson M, Soderpalm B (2015). Nicotine produces chronic behavioral sensitization with changes in accumbal neurotransmission and increased sensitivity to re-exposure. Addict Biol 21: 397-406.

Murray JE, Belin D, Everitt BJ (2012). Double dissociation of the dorsomedial and dorsolateral striatal control over the acquisition and performance of cocaine seeking. Neuropsychopharmacology 37: 2456-2466.

Nisell M, Nomikos GG, Svensson TH (1994a). Infusion of nicotine in the ventral tegmental area or the nucleus accumbens of the rat differentially affects accumbal dopamine release. Pharmacol Toxicol 75: 348-352.

Nisell M, Nomikos GG, Svensson TH (1994b). Systemic nicotineinduced dopamine release in the rat nucleus accumbens is regulated by nicotinic receptors in the ventral tegmental area. Synapse 16: 36-44.

Pontieri FE, Tanda G, Orzi F, Di Chiara G (1996). Effects of nicotine on the nucleus accumbens and similarity to those of addictive drugs. Nature 382: 255-257.
Ren Z, Sun WL, Jiao H, Zhang D, Kong H, Wang X et al (2010). Dopamine D1 and $N$-methyl-D-aspartate receptors and extracellular signal-regulated kinase mediate neuronal morphological changes induced by repeated cocaine administration. Neuroscience 168: 48-60.

Schoffelmeer AN, De Vries TJ, Wardeh G, van de Ven HW, Vanderschuren LJ (2002). Psychostimulant-induced behavioral sensitization depends on nicotinic receptor activation. J Neurosci 22: 3269-3276.

Sommer WH, Costa RM, Hansson AC (2014). Dopamine systems adaptation during acquisition and consolidation of a skill. Front Integr Neurosci 8: 87.

Takahashi Y, Roesch MR, Stalnaker TA, Schoenbaum G (2007). Cocaine exposure shifts the balance of associative encoding from ventral to dorsolateral striatum. Front Integr Neurosci 1: 11.

Vezina P, McGehee DS, Green WN (2007). Exposure to nicotine and sensitization of nicotine-induced behaviors. Progr Neuropsychopharmacol Biol Psychiatry 31: 1625-1638.

Volkow ND, Wang GJ, Telang F, Fowler JS, Logan J, Childress AR et al (2006). Cocaine cues and dopamine in dorsal striatum: mechanism of craving in cocaine addiction. J Neurosci 26: 6583-6588.

Voorn P, Vanderschuren LJ, Groenewegen HJ, Robbins TW, Pennartz CM (2004). Putting a spin on the dorsal-ventral divide of the striatum. Trends Neurosci 27: 468-474.

Wickens JR, Reynolds JN, Hyland BI (2003). Neural mechanisms of reward-related motor learning. Curr Opin Neurobiol 13: 685-690.

Willuhn I, Burgeno LM, Everitt BJ, Phillips PE (2012). Hierarchical recruitment of phasic dopamine signaling in the striatum during the progression of cocaine use. Proc Natl Acad Sci USA 109: 20703-20708.

Yan Y, Pushparaj A, Le Strat Y, Gamaleddin I, Barnes C, Justinova $\mathrm{Z}$ et al (2012). Blockade of dopamine $\mathrm{d} 4$ receptors attenuates reinstatement of extinguished nicotine-seeking behavior in rats. Neuropsychopharmacology 37: 685-696.

Yin HH, Knowlton BJ, Balleine BW (2006). Inactivation of dorsolateral striatum enhances sensitivity to changes in the action-outcome contingency in instrumental conditioning. Behav Brain Res 166: 189-196.

Yin HH, Mulcare SP, Hilario MR, Clouse E, Holloway T, Davis MI et al (2009). Dynamic reorganization of striatal circuits during the acquisition and consolidation of a skill. Nat Neurosci 12: 333-341.

Yin HH, Ostlund SB, Balleine BW (2008). Reward-guided learning beyond dopamine in the nucleus accumbens: the integrative functions of cortico-basal ganglia networks. Eur J Neurosci 28: 1437-1448.

Yin HH, Ostlund SB, Knowlton BJ, Balleine BW (2005). The role of the dorsomedial striatum in instrumental conditioning. Eur $J$ Neurosci 22: 513-523.

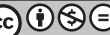

This work is licensed under a Creative Commons Attribution-NonCommercial-NoDerivs International License. The images or other third party material in this article are included in the article's Creative Commons license, unless indicated otherwise in the credit line; if the material is not included under the Creative Commons license, users will need to obtain permission from the license holder to reproduce the material. To view a copy of this license, visit http://creativecommons.org/licenses/by-nc-nd/4.0/

(C) The Author(s) 2016 